\title{
Gene editing: how to stay on-target with CRISPR
}

\author{
Vivien Marx
}

Efficiently cutting a target sequence to effect a desired change in the genome is one gene-editing task. Knowing where else in the genome a tool might have made its mark is quite another.

Bacteria practice gene editing, and human genome engineers have taken notice. To thwart invading viruses, bacteria snip some of the invader's nucleic acid out of their genome using a special scissors: a nuclease. The bacterium keeps a genetic memory of the invader at a locus where viral DNA is interspersed with bacterial DNA. This locus, clustered, regularly interspaced, short palindromic repeats (CRISPR), is then used to transcribe RNAs that specifically target a nuclease to viral DNA during future infections.

One version of this bacterial immune defense-CRISPRs associated with the nuclease Cas9-has been turned into a lab tool $^{1}$ that captivates the interest of academic and commercial researchers. The CRISPRCas RNA-guided system mainly pairs guide RNA (gRNA) with the target site to be edited. It shows versatile use in many organisms and allows modifications to multiple target sites simultaneously. Accompanying this technique's rise to popularity is an intensi-

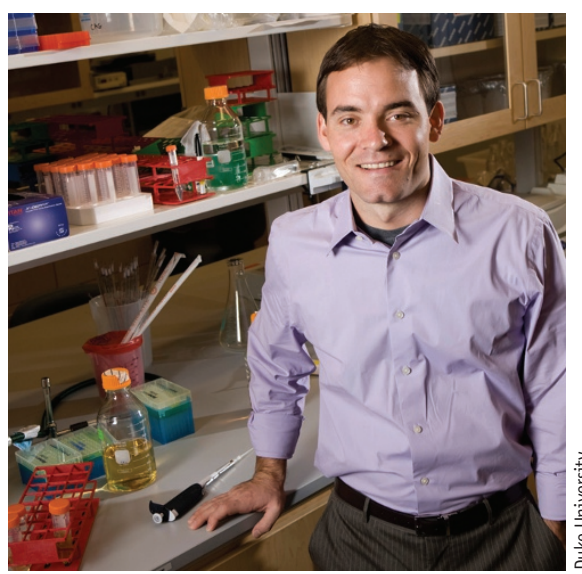

Early CRISPR work probably looked at the worstcase scenarios, with experimental conditions favorable for off-target effects, says Charles Gersbach.

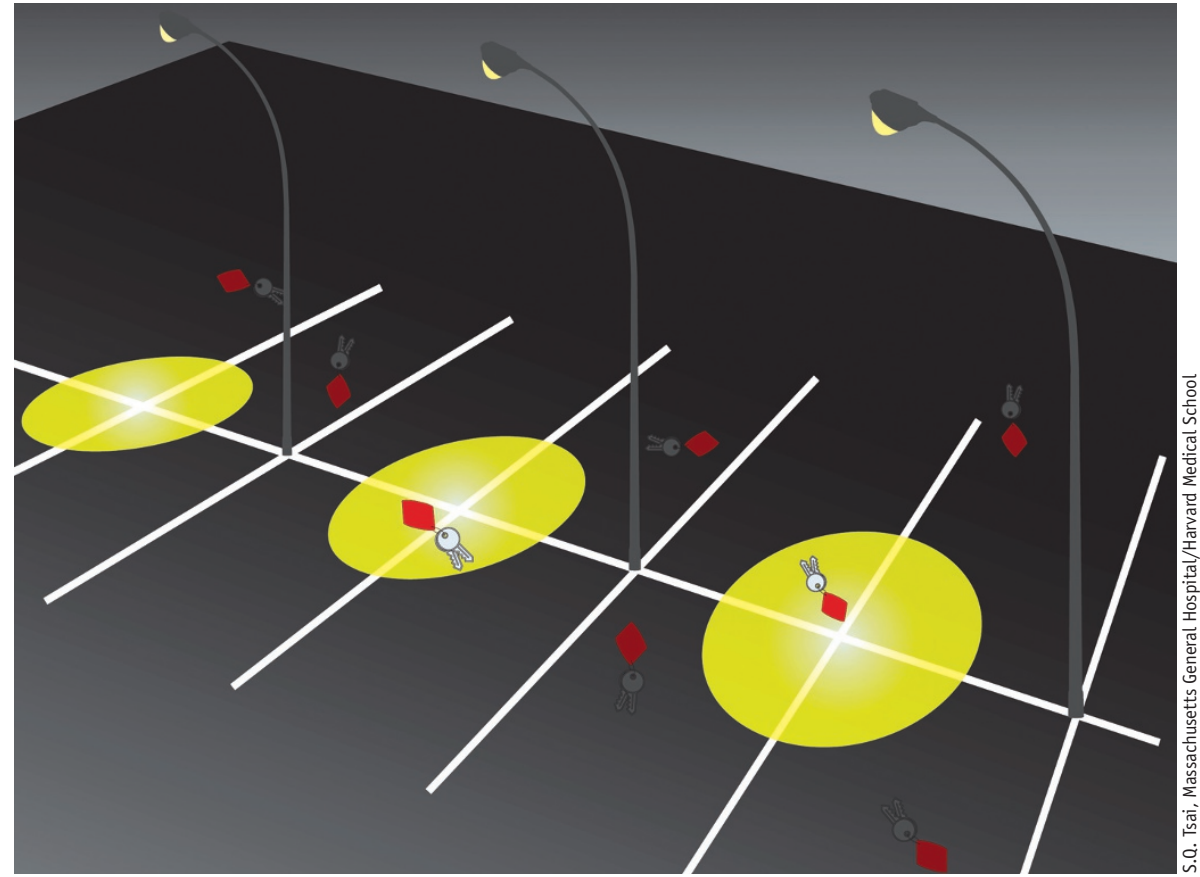

There are assays to predict and assess off-target effects, but for now they offer only partial illumination of the genome.

fying discussion: how to edit the genomic location of interest and reduce so-called offtarget effects, which are unintended edits elsewhere in the genome.

Gene editing leverages the cells' DNA repair mechanisms: nonhomologous end joining (NHEJ) or homologous recombination. Particularly when NHEJ is the repair mode, unwanted sequence changes can occur at both on- and off-target sites. Too many off-target breaks can be toxic to cells, and they can lead to structural rearrangements of the genome as the breaks are repaired. One fear is the risk of activating oncogenes $^{2}$.

Scientists interviewed by Nature Methods say that too few are aware of the risks that such off-target effects pose and of the emerging methods to reduce the frequency with which they happen. Speaking anonymously, one researcher says there is a lack of sufficient controlled comparative data on the "true specificity properties" of various proposed nuclease architectures.

In some but not all experiments, off-targets can be mission critical. New assessment methods will help, says Charles Gersbach, a biomedical engineer at Duke University. Predicting off-target sites depends on the target sequence to be edited. Of the many techniques to measure or address specificity, none is "perfect," says Gersbach. "However, I do not think that there is any data published thus far that indicates CRISPR is fundamentally limited by specificity issues," he says.

With CRISPR-Cas, activity at the target site is usually found in between $1 \%$ and $10 \%$ of transfected cells, he says, and off-target 


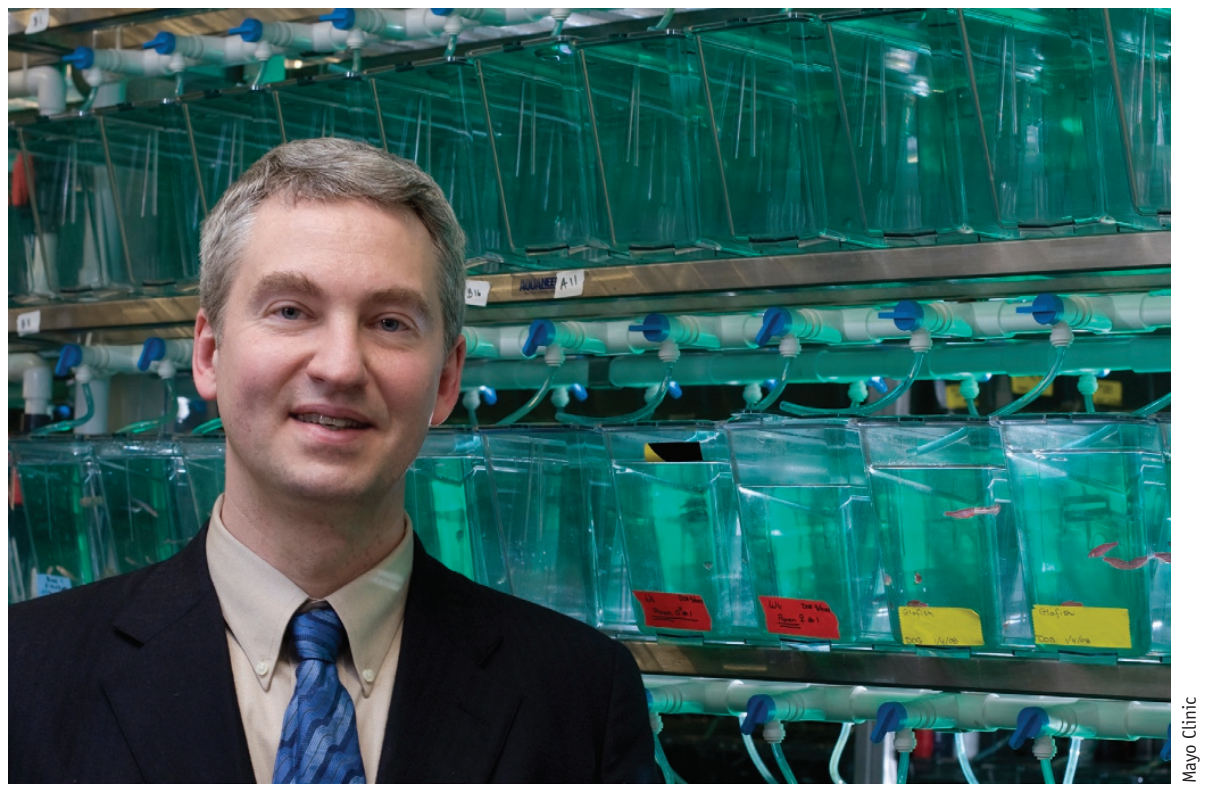

Experience with established tools offers lessons about off-target issues, says Stephen Ekker.

activity is "significantly lower." There are only a few off-target sites across the whole genome, with low activity at those sites. Recent studies-for example, in edited induced pluripotent stem cells-showed no measurable off-target effects, he says. Offtarget edits typically add or remove a few base pairs, which makes it possible to determine whether the changes might be problematic.

Gersbach contrasts these approaches to gene therapy strategies with viral vectors, which can integrate randomly in the genome and which sometimes show preference for gene-coding regions. They are "significantly more disruptive than the potential CRISPR-Cas9 off-target effects." And, he says, several approved cancer therapies disrupt the genome more than the reported CRISPR-induced off-targets.

Early CRISPR work probably looked at the worst-case scenarios, such as experimental conditions "highly favorable for off-target effects, and even then, overall off-target frequencies were relatively low," he says.

For example, scientists used immortalized cell lines such as HEK293 that have a number of genomic abnormalities and a deregulated cell cycle that affects DNA replication. The cells also express high levels of Cas9 and gRNA. "It is well established that off-target effects increase with greater levels of Cas9 and gRNA," he says.

$\mathrm{He}$ and his team are currently using CRISPR-Cas9 to correct mutations that cause Duchenne muscular dystrophy. To probe for off-target effects and to ascertain their possible biological impact, the group is comparing the off-target effects in HEK293 cells and in patient myoblasts.

\section{Lessons from history}

Experience with established tools offers lessons about off-target issues, says Stephen Ekker, a molecular biologist at the Mayo Clinic, noting experiments with chemical mutagenesis, RNA interference screening, antisense RNAs, and insertional mutagenesis using viruses and transposons, as well as work focused on zinc-finger nucleases (ZFNs) and transcription activator-like effector nucleases (TALENs).

Off-targeting is a potential confounding variable that can confuse experimental outcome when these tools are used, says Ekker. "At worst, off-target cutting is more efficient than on-target changes," he says. Some effects can be predicted, and some can happen unexpectedly; experimental design can help address both.

One approach in his lab is to engineer zebrafish with allelic modifications using different gRNAs for CRISPR-Cas9 mutagenesis. First-generation zebrafish carrying a CRISPR-Cas9-modified allele might have enough other changes that the animals need to be outcrossed to distinguish targeted mutations linked to phenotypes of interest from those caused by off-target effects. With cells, however, this outcrossing is not possible. And off-target effects can also be hard to disentangle from the genomic scramble already present in cancer cells growing in a dish. 
Gene-editing tools differ in their ability to target a unique sequence in the vertebrate genome, says Ekker. Whereas ZFNs and TALENs tend to have sufficient specificity for the task, the standard CRISPRCas system does not. Scientists will want to choose the tool that best answers their scientific question. "Hammers are great, but so are screwdrivers and so are pliers," he says. Now that a "CRISPR mindshare" is in place, a large number of labs are learning more broadly about genome engineering tools and approaches.

\section{Practical considerations}

Whereas gene-editing techniques such as ZFNs and TALENs use protein-DNA interactions to edit a location in a genome, the CRISPR-Cas RNA-guided system pairs gRNA sequence with DNA at the target site. One advantage of this RNA-driven recognition is that it is easier for a nonspecialist to use, says Keith Joung, a molecular biologist at Massachusetts General Hospital. He is also scientific cofounder and consultant with Editas, a company seeking to apply gene editing therapeutically. The protein-DNA interactions typical for ZFNs, TALENs or meganucleases are more complex.

Compared to the other gene-editing tools, CRISPR makes it easier to target a single gene or region multiple times by using a number of gRNAs. It is also easier, he says, to generate multiple mutations at the same time in a single cell.

In their experiments, some scientists alter the concentrations of gRNA and Cas9 used, says Yuichiro Miyaoka, a postdoctoral fellow in Bruce Conklin's lab at the Gladstone Institute of Cardiovascular Disease. He says the highest specificity comes with the lowest Cas 9 and gRNA plasmid dosage. But the lowest dosage can also lower on-target activity. Some groups have seen reductions in off-target effects without compromised on-target efficiencies, whereas others have observed reductions in both on- and offtarget effects, suggesting that the effect of this approach may vary from sequence to sequence, says Joung.

Some scientists suggest using rational design or directed evolution, or a com- bination of both, to improve upon the RNA-guided Cas9 protein with desired characteristics ${ }^{3}$. Other groups shorten the time during which Cas9 is active. But experimenters will not want to shorten the window so much that on-target efficiency is also reduced, says Joung.

In CRISPR-Cas-based experiments, target sequence selection is important: the goal is to predict the ones-which can be sequences within a gene-with the least number of potential off-target effects. Although the approach "intuitively makes sense," says Joung, what is unclear is how dissimilar or mismatched the off-target sites might need to be.

He and his team have shown with wildtype Cas9 that some off-target effects happen at sites that differ by up to five bases. For any given on-target site, there may be thousands of sites with up to five mismatches, and it is not evident which will be cleaved and which will not. "So although picking sites with fewer mismatched sites can't hurt, it isn't clear to me that this strategy actually reduces your off-target effects," he says. 


\section{BOX 1 OFF-TARGET PREDICTION TOOLS}

Many software tools predict potential off-target effects for gene-editing tools. Gang Bao, a biomedical engineer from Emory University and Georgia Institute of Technology, believes that there is not yet enough published experimental data to evaluate and validate these tools. These data will help train algorithms with machine learning so that they can better predict and rank potential off-target sites.

Predictions with existing tools can mean many false positives and false negatives. "Therefore, there are a lot of reasons to advise caution," he says, and to experimentally validate all results.

Plenty of potential off-target effects can be missed also because the tools consider base mismatches between gRNA and the target but leave out DNA or RNA bulges, which are damaged sections in which some mismatched bases bulge out, says Bao. He has a new prediction tool in the works that includes bulges. Below is a noncomprehensive list of tools.

Cas0T (http://eendb.zfgenetics.org/casot/)

PKU Zebrafish Functional Genomics group, Peking University

CHOPCHOP (https://chopchop.rc.fas.harvard.edu/)

Harvard University

CRISPR Design (http://crispr.mit.edu/)

Feng Zhang, Massachusetts Institute of Technology

CRISPR Design tool (http://www.broadinstitute.org/mpg/crispr_design/)

The Broad Institute of Harvard and MIT

CRISPR/Cas9 gRNA finder (http://spot.colorado.edu/ slin/cas9.html)

Jack Lin, University of Colorado

CRISPRfinder (http://crispr.u-psud.fr/Server/)

Christine Pourcel, Université Paris-Sud 11

E-CRISP (http://www.e-crisp.org/E-CRISP/)

DKFZ German Cancer Research Center

CRISPR gRNA Design tool (https://www.dna20.com/eCommerce/cas9/input)

DNA 2.0

PROGNOS (http://baolab.bme.gatech.edu/cgi-bin/prognos/prognos.cgi)

Gang Bao, Emory University/Georgia Institute of Technology

ZiFiT (http://zifit.partners.org/ZiFiT)

Keith Joung, Massachusetts General Hospital

A set of approaches addresses off-target effects by altering the architecture of gRNAs. Truncated gRNAs ${ }^{4}$ are a little counterintuitive, says Joung, but this shortening may reduce the gRNAs' binding energy, making them more sensitive to mismatches at the gRNA-DNA interface and therefore improving specificity. The truncation of gRNAs reduces the frequency of off-target effects at some sites by a factor of 5,00010,000 or more, he says. In contrast, other groups have shown the opposite, namely that adding two Gs at the end of the gRNA reduces off-targets ${ }^{2}$.

Researchers can also use paired gRNAs or Cas9 nickases, which create single-strand nicks as opposed to the double-strand break caused by nucleases. In this strategy, two nicks are placed close to one another and on opposing strands of DNA to create essentially a double-strand break. This strategy has been shown to reduce the off-target effects of a single Cas9 nuclease, says Joung. But this system relies on colocalization rather than dimerization of monomeric Cas9 nickases, which remain enzymatically active and can induce insertions, deletions and point mutations at certain sites.

Another possibility is to apply CRISPR RNA-guided Fok1 nucleases (RFNs), which use the dimerization-dependent FokI nuclease domain that is also used for ZFN and TALEN applications. And it requires two appropriately positioned gRNA-RFN complexes to achieve DNA cleavage. In contrast to Cas9 nickases, RFNs show minimal, if any, activity as monomers, says Joung ${ }^{4}$. All of these approaches can potentially be combined in different ways, too.

\section{Predictions and assays}

It is also possible to draw on computational tools to find off-targets (Box 1). However, a researcher who wishes to remain anonymous tells Nature Methods that a comparison of predicted off-target 
effects and published results from an unbiased screen with CRISPR-Cas9 led to data sets with "very little overlap." Commenting on this anecdote, Miyaoka says it is a reminder that computational tools are imperfect.

Miyaoka and colleagues computationally predict off-target sites and analyze these with Sanger sequencing or the CEL I mutation detection assay, which uses a mismatch-specific endonuclease from celery to find single-nucleotide polymorphisms and short insertions or deletions. The T7 endonuclease 1 assay is another kind of mismatch assay. Wholegenome sequencing is Miyaoka's favorite assay, but sequencing all clones is not feasible. The best assay is "yet to be determined," he says.

Digital PCR could be helpful as a quantitative assay for rare genome-editing events, says Miyaoka. For example, different tools and gene-editing conditions can slant DNA repair mechanisms toward or away from homologous recombination or NHEJ. Conklin and he are working on ways to measure this balance using digital PCR.

The CEL- 1 assay is also available as the Surveyor Mutation Detection kit from Transgenomic. In early July, Transgenomic sold rights to this assay to Integrated DNA Technologies (IDT). Scientists working in cell lines or model organisms in basic research or for preclinical drug discovery can obtain the assay through IDT. If they are using this assay in CRISPR-based therapies, they will need to obtain the assays, which are aimed at clinical targets, from Transgenomic, a company spokesperson says.

There is currently no perfect way address specificity or to comprehensively measure it in every modified cell, says Gersbach. This situation makes it difficult to gauge how problematic specificity is or the degree to which a method improves specificity.

When researchers are modeling disease in cells or generating genetically modified organisms, they can sequence the whole genome of a clonal cell population or organism. "However, this can be tedious to do routinely, and it is not applicable to cells that cannot be clonally derived, which includes all the cell types currently being pursued clinically for gene therapies," says Gersbach. Advances in highthroughput sequencing and in single-cell analysis indicate that better techniques to assess specificity are on the horizon.

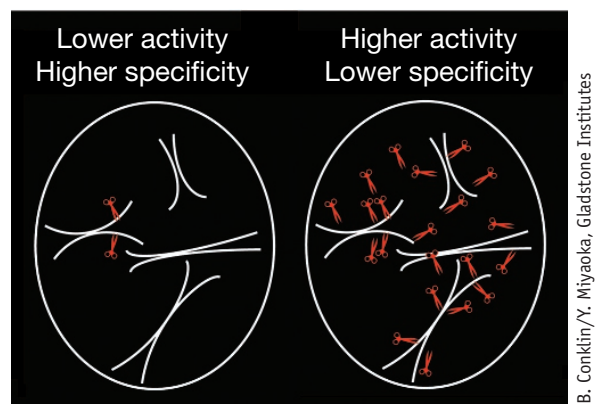

In genome editing, reducing off-target effects is a calculated trade-off. With a highly active nuclease, unintentional breaks in the genome are more likely.

\section{Validated reagents}

CRISPR-Cas has been harnessed for geneediting experiments in many organisms to create cell lines and genetically engineered animals. There are now several companies that let researchers submit a gene, and then the company sends gRNAs. But these still need to be validated in the lab. Chris Kubu, a researcher at Affymetrix, recommends using array profiling to assess exon-level gene expression information or to see subtle changes in gene isoforms at the end of a gene-editing experiment.

Companies such as Horizon Discovery, Sage Labs, Sigma-Aldrich, Thermo Fisher Scientific and ToolGen offer reagents for CRISPR-Cas-based experiments, cell lines and animals. Sage Labs has begun selling SAGEspeed CRISPR reagents, which are used within the company to create animal models. When a customer orders a reagent, the company builds and evaluates five designs and tests cutting efficiency in a cell-based assay to choose the best one, says Kevin Gamber, who directs the company's marketing and business development.

The team uses bioinformatics tools to design gRNAs. Given that the longer CRISPR-Cas is in the cell, the more likely an off-target event becomes, the company also limits the reagents' half-lives, says Gamber. And Sage uses CRISPR-Cas9 as RNA rather than plasmid systems because RNA is more quickly degraded than plasmids, he says.

The company has a tiered validation approach. Researchers can obtain unvalidated reagents. They can also choose SAGEspeed reagents with tested designs and validated nucleases. Another option, 'Validated+' reagents, comes with primers that target the top ten predicted off-target sites for the gRNA. Scientists can also have the company perform the assays for them. 


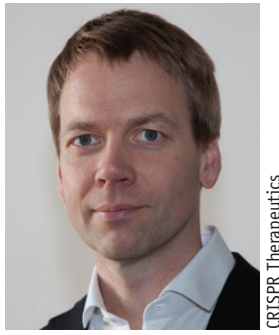

The CRISPR-Cas

system can have

“highly, highly

effective" on-target

activity, says Rodger

Novak.

ZFN-based origins.

To edit a cell line or model organism's genome, the Sage Labs team might choose ZFNs, which target 24-34 base pairs as opposed to CRISPR's 20-basepair target. In Gamber's view, some of the modifications to the CRISPR-Cas9 system to reduce offtarget effects have

At Sigma-Aldrich, most CRISPRs are not validated in cell-based assays, but the company uses computational tools to design the most specific CRISPRs, says staff scientist Greg Davis. His team develops gene-editing tools with in-house and in-licensed approaches. They use paired nickases, for which they have filed patents, when computational tools offer too few options. A core requirement is that all gRNAs differ from all other genomic sites by at least three base pairs. "This requirement is relaxed in some difficult situations, but always in close collaboration with the customer to weigh comfort levels," he says.

Prediction of off-target potential is based on published genome sequences, but individual variability can mean that off-target activity will vary from one cell line to another, says Chris Thorne, who develops Horizon Discovery's gene-editing reagents and cell lines.

When producing an edited cell line with CRISPR-Cas9, customers can opt to have the five most likely off-target sites sequenced in multiple independent clones, as predicted by the company's platform, called gUIDEbook, which looks for sequence homologies. Whole-exome or whole-genome sequencing are options, "but both the cost and analytical work involved are prohibitive at this stage," he says.

Horizon scores gRNAs for off-target potential during the design stages, and only top scorers that show cutting activity in cell-based assays progress to further validation. He notes that not all guides mediate cutting with equal efficiency, and the parameters of influence are a subject of ongoing research, he says. One of Horizon's assays is Surveyor, which delivers a "semiquantitative assessment" of cutting activity in a pool of transfected cells.

In July, the company began offering five gRNAs for free to any academic lab knocking out a gene in human cells. Researchers can obtain gRNAs for up to five genes, with five gRNAs per gene. In return, the scientists share data on the activity of these gRNAs with Horizon, which feeds back into the company's design platform, he says. The scientists license the cell lines they create back to Horizon and can earn royalties.

\section{Outlook}

A number of companies position themselves for applications in people, such as CRISPR Therapeutics and Editas, a goal that heightens the importance of techniques to predict and assess off-target gene editing. One industry researcher who does not wish to be identified says it is likely that applications will involve patients who have few or no other options, in which case the "putative risks of off-target nuclease activity will be overwhelmed by the potential benefit to patients."

Sangamo BioSciences is sponsoring a clinical trial in which T cells of HIV-positive patients are removed, the genomes are edited with ZFNs and the cells are infused back into patients. The idea is that the edited, disrupted CCR5 gene, which encodes a receptor on the CD4 T-cell surface, can make the T cells less susceptible to HIV. Of the 12 patients, 6 have been able to interrupt their traditional antiviral treatment ${ }^{5}$.

Sangamo is targeting cells that are readily accessible and is editing a scientifically well-understood system, one in which clinicians also have much experience, says Rodger Novak, CEO of CRISPR Therapeutics. "There are a number of boxes that have been ticked," he says.

For other applications, such as potentially editing genes in organ systems in vivo, the same rules will hold. "We need to understand the target," says Novak, who previously worked at the pharmaceutical company Sanofi.

The CRISPR-Cas system cuts DNA efficiently, and it can have "highly, highly effective" on-target activity, he says, which are prerequisites to advancing a potential therapeutic. And when the right target is chosen, it might not be necessary to dramatically boost the on-target activity of the CRISPR-Cas system.

What is needed, he says, are assays that let researchers assess risks and know about both off-target issues and the overall effect of CRISPR-Cas gene editing on a cell. It means understanding what determines cutting quality, finding out which off-targets matter in a given clinical situation and having benchmarks for comparison. "If I try to improve the technology today, I don't know against what do I improve it," says Novak.

Assessing off-target effects, such as cell toxicity, genotoxicity and safety, is part of daily screens in drug development, which suggests how gRNAs could be screened on a larger scale. When the safety levels for a given gRNA are good, tinkering with it might reduce on-target activity and could end a potential therapeutic's development. In other cases, reduced on-target activity might be needed for a given clinical application. "It's a case-by-case decision," says Novak.

No single genome-editing reagent has everything: none is accessible, has the specificity researchers want and lets them target with sequence flexibility, too, says Ekker. CRISPR-Cas is the most accessible system, in his view, and the least specific. And using more specific dual CRISPR-Cas9 systems can mean losing the ability to target sequence flexibly.

As Joung explains, what has not yet been described in the research literature is a sensitive, unbiased and genome-wide way to assess potential off-target effects so that researchers can identify nucleases with the fewest number of deleterious offtarget effects. Over time, many technological developments have improved CRISPR specificity, and as CRISPR's popularity continues, more approaches are bound to follow.

1. Jinek, M. et al. Science 337, 816-821 (2012).

2. Cho, S.W. et al. Genome Res. 24, 132-141 (2014).

3. Mali, P., Esvelt, K.M. \& Church, G.M. Nat. Methods 10, 957-963 (2013).

4. Fu, Y., Sander, J.D., Reyon, D., Cascio, V.M. \& Joung, J.K. Nat. Biotechnol. 32, 279-284 (2014).

5. Tebas, P. et al. N. Engl. J. Med. 370, 901-910 (2014).

Vivien Marx is technology editor for Nature and Nature Methods

(v.marx@us.nature.com). 